\title{
MS07-P08 | NEW STRUCTURAL INSIGHT INTO THE WELL KNOWN PEPTIDE FLIP OBSERVED IN
}

\section{FLAVODOXINS.}

Gudim, Ingvild (University of Oslo, Oslo, NOR); Lofstad, Marie (University of Oslo, Oslo, NOR); Hammerstad, Marta (University of Oslo, Oslo, NOR); van Beek, Wouter (European Synchrotron Radiation Facility, Grenoble, FRA); Hersleth, Hans-Petter (University of Oslo, Oslo, NOR)

Flavodoxins (Flds) are small proteins that shuttle electrons in a range of reactions in microorganisms. Flds contain a redox-active cofactor, a flavin mononucleotide (FMN), and it is well established that when Flds are reduced by one electron, a peptide bond close to the FMN isoalloxazine ring flips to form a new hydrogen bond with the FMN N5H, stabilising the one-electron reduced state. Here, we present high-resolution crystal structures of Flavodoxin 1 from Bacillus cereus in both the oxidised and one-electron reduced (semiquinone) state. We observe a mixture of conformers in the oxidised state; a 50:50 distribution between the established oxidised conformation where the peptide bond is pointing away from the flavin, and a conformation where the peptide bond is pointing toward the flavin, approximating the conformation in the semiquinone state. We use single-crystal spectroscopy to demonstrate that the mixture of conformers is not caused by radiation damage to the crystal. This is the first time that such a mixture of conformers is reported in a wild-type Fld. We therefore carried out a survey of published Fld structures, which show that several proteins have a pronounced conformational flexibility of this peptide bond. The degree of flexibility seems to be modulated by the presence, or absence, of stabilising interactions between the peptide bond carbonyl and its surrounding amino acids [1,2].

[1] I. Gudim, M. Lofstad, W. van Beek, H.-P. Hersleth, Protein Sci. 27 (2018) 1439-1449.

[2] I. Gudim, M. Hammerstad, M. Lofstad, H.-P. Hersleth, Biochemistry 57 (2018) 5427-5436. 